\title{
МОДЕЛИРОВАНИЕ ДИФФУЗИИ ПОКОЛЕНИЙ ТЕХНОЛОГИЙ МОБИЛЬНОЙ СВЯЗИ
}

\author{
Дубинина М.Г.
}

Статья посвящена анализу технико-экономических показателей поколений технологий мобильной связи, выявлению факторов, влияющих на их появление и распространение. Разработаны модели диффузии сетей $2 G, 3 G$ и $4 G$ по странам ОЕСD и России за период 2000-2018 г2. с учетом влияния сопутствуюших технологий (интернета и мобильного широкополосного доступа в интернет) и взаимного влияния предшествующего и последующего поколений на скорость и масштаб распространения каждого вида сети.

DOI: $10.20537 /$ mce2020econ09

Введение. С момента своего появления в начале 1980-х гг. система мобильной связи прошла несколько этапов развития, совершенствуя свои стандарты для удовлетворения постоянно меняющегося спроса на мобильные услуги по всему миру. В результате многочисленных изменений, которые произошли в технологиях мобильной связи, стали выделять их поколения $(\mathrm{G})$, характеризующиеся различными функциональными возможностями работы сети (скоростью, частотой, емкостью данных, задержкой сигнала), способами передачи информации, набором услуг, предоставляемых абоненту. Существующие методы и модели прогнозирования показателей техники и технологии основаны на генетическом анализе, который позволяет выделять среди перечня параметров, описывающих данный вид технологии (техники), главный и основные (см. $[1,2,3])$. Далее представлен такой анализ поколений технологий мобильной связи.

Технико-экономические характеристики поколений технологий мобильной связи. Поколение $1 \mathrm{G}$ появилось в 1982 г., было аналоговой связью, технология использовалась только для голосовых вызовов и предполагала множественный доступ с пропускной способностью канала 30 кГц и полосой частот 824-894 МГц [4]. Недостатками поколе-

\footnotetext{
* Работа выполнена при частичной поддержке гранта РФФИ №20-010-00065.
} 
ния $1 \mathrm{G}$ были низкая скорость, плохое качество передачи звука, малое время автономной работы первых сотовых телефонов, их большой размер, ограниченные возможности и низкий уровень эффективности использования спектра. При этом отсутствовала защита связи.

Поколение $2 \mathrm{G}$ использует цифровые сигналы для передачи голоса, текстовых и графических сообщений на низкой скорости в полосе пропускания от 30 до 200 кГц. Сети $2 \mathrm{G}$ обеспечили почти 25-кратное увеличение скорости по сравнению с $1 \mathrm{G}$. С появлением интернета мобильные сети стали характеризоваться более высокой пропускной способностью передачи данных через GPRS (General Packet Radio Service).

Поколение 3G разрабатывалось для предоставления высокоскоростной передачи данных с использованием пакетов переключения, которая обеспечила увеличенную емкость канала и поддержку мультимедиа, предоставила услуги глобального роуминга, доступа к телевидению, видео. Потребность в мобильной широкополосной связи (3G и выше) еще более возрастает в связи с повсеместным распространением новых мобильных устройств (смартфонов, планшетов, нетбуков), с ростом числа предприятий, правительственных учреждений, служб экстренной помощи, использующих данную технологию.

Поколение 4G является развитием мобильных технологий и преодолевает ограничения $3 \mathrm{G}$, увеличивая пропускную способность и снижая стоимость ресурсов [5]. Эта технология мобильной связи предоставляет, помимо функций $3 \mathrm{G}$, дополнительные услуги (например, просмотр ТВ-программ высокой четкости, цифровое видеовещание и др.) при низкой стоимости за бит информации, однако для ее реализации требуется сложное оборудование. До сих пор в некоторых странах (особенно развивающихся) технологии $4 \mathrm{G}$ находятся на ранней стадии внедрения. Развивающиеся страны больше внимания уделяют развертыванию $3 \mathrm{G}$ (охват 4G в среднем составляет 35\%). Существенным исключением является Китай, достигший охвата технологией $3 \mathrm{G}$ в $99 \%$ менее чем за три года и такими же темпами внедривший технологии 4G.

В начале 2010-х гг. началась разработка сетей поколения 5G, которые представляют собой следующий значительный скачок в мобильной связи с обещанными скоростями для пользователей, приближающимися к 1 Гбит/с и задержкой сигнала в 1 мс. Ожидается, что в сетях 5G большой объем полосы пропускания поможет удовлетворить спрос на потребление мобильных данных, которое прогнозируется от увеличения подписчиков мобильной связи до примерно 1 млрд человек в 2023 г. [6]. 
Переход на $5 \mathrm{G}$ будет включать улучшения существующей инфраструктуры и добавление нового фрагмента спектра в диапазоне от 24 ГГц до 90 ГГц. На начало 2020 г. сети $5 \mathrm{G}$ полноценно функционировали только в США, Южной Корее и Швейцарии. В январе 2020 г. в России была запущена первая промышленная сеть 5G на заводе «Камаз» [7].

Основные технические характеристики поколений мобильной связи показаны в табл. 1.

Таблица 1. Основные показатели поколений технологий мобильной связи (по данным $[8,9,10])$

\begin{tabular}{|l|c|c|c|c|c|}
\hline Показатель & $\mathbf{1 G}$ & $\mathbf{2 G}$ & $\mathbf{3 G}$ & $\mathbf{4 G}$ & $\mathbf{5 G}$ \\
\hline Год начала разработки & 1970 & 1980 & 1990 & 2000 & 2010 \\
\hline Год внедрения & 1984 & 1991 & 2001 & 2009 & 2019 \\
\hline $\begin{array}{l}\text { Средняя скорость загрузки, } \\
\text { Мб/сек }\end{array}$ & 0.0024 & 0.064 & 2 & 300 & 10000 \\
\hline Пиковая скорость, Мб/сек & 0.0144 & 0.144 & 42 & 1024 & 10480 \\
\hline Средняя задержка, мс & $\ldots$ & 500 & 150 & 50 & 1 \\
\hline $\begin{array}{l}\text { Поддержка мобильности, } \\
\text { км/ч }\end{array}$ & $\ldots$ & $\ldots$ & 300 & 400 & 500 \\
\hline Ширина полосы, Мб/с & 0.002 & 0.064 & 2 & 200 & $>1000$ \\
\hline Радиус соты, км & 20 & 30 & 35 & 80 & $\ldots$ \\
\hline Трафик, Эб/мес. & 0 & 0.78 & 2.35 & 8.05 & 50 \\
\hline $\begin{array}{l}\text { Спектральная эффективность } \\
\text { бс/Гц }\end{array}$ & $\ldots$ & 0.17 & 0.75 & 1.5 & $>3$ \\
\hline $\begin{array}{l}\text { Средняя выручка на 1 под- } \\
\text { писчика (2012 г., долл./мес.) }\end{array}$ & 8 & 6 & 25 & 64 & $50 *$ \\
\hline
\end{tabular}

* оценка на 2021 г.[11]

Показатель спектральной эффективности, который вычисляется как отношение скорости передачи данных к ширине полосы пропускания, может служить оценкой эффективности использования частотного диапазона поколения технологий мобильной связи [12].

Определение средней цены по поколению технологии мобильной связи достаточно сложно, однако, согласно исследованию [13], в период 2005-2013 гг. средняя стоимость мобильного тарифа за мегабайт данных снизилась на 99\%, затраты на инфраструктуру мобильной связи для сетей $3 \mathrm{G}$ сократились на $95 \%$ по сравнению с $2 \mathrm{G}$ (в расчете на мегабайт 
передаваемых данных), а для 4G они сократились на 67\% по сравнению с сетями $3 \mathrm{G}$.

Все цифровые технологии мобильной связи в настоящее время сосуществуют. Однако в ряде стран уже ликвидированы сети 2G (Япония, Макао, Сингапур, Южная Корея), в то же время в европейских странах широкое развертывание сервисов межмашинного взаимодействия (M2M, machine-to-machine) и интернета вещей (IoT), основанных на технологии $2 \mathrm{G}$, может привести к более быстрому отключению сетей $3 \mathrm{G}$ по сравнению с $2 \mathrm{G}$ [14]. В России в настоящее время около $15 \%$ мобильных устройств поддерживают только сети $2 \mathrm{G}$ (кнопочные телефоны, детские часы, различные М2М-датчики, спутниковые трекеры и т.д.), кроме того, большое количество систем аварийной сигнализации основаны на GSM-модемах [15].

Современная тенденция быстрого роста объемов трафика компьютерных сетей обусловлена развитием таких технологий, как облачные вычисления, облачные хранилища, «потоковые» сервисы для фильмов, музыки или игр. За период 2010-2017 гг. объем общего IP-трафика увеличился с 20.4 Эб/месяц в 2010 г. до 122 Эб/месяц в 2017 г. (рост почти в 6 раз), тогда как мобильный трафик увеличился в 50 раз (с 0.24 Эб/м до 12 Эб/м соответственно), его доля в общем IP-трафике выросла с $1.2 \%$ в 2010 г. до $9.8 \%$ в 2017 г. [16, 17].

Моделирование взаимного влияния поколений сотовой связи на их распространение. В данной работе исследуется взаимное влияние поколений технологий мобильной связи, а также факторы, которые влияют на их распространение. Исследование проведено по данным за 2000-2018 гг. из отчетов и баз данных WorldBank, OECD, Росстата и Евросоюза по странам OECD и России. В качестве основы исследования была использована модифицированная модель Нортона-Басса [18] для описания диффузии нескольких поколений технологий с учетом влияния сопутствующих технологий вида: 


$$
\left\{\begin{array}{l}
\frac{d g_{2}(t)}{d t}=\left(p_{1}+\frac{q_{11}}{M_{1}} g_{2}(t)+\frac{q_{12}}{M_{1}}(m p h(t)-) g_{2}(t)\right)\left(M_{1}-g_{2}(t)\right)+a_{1} \operatorname{int}(t) \\
\frac{d g_{3}(t)}{d t}=\left(p_{2}+\frac{q_{21}}{M_{2}} g_{3}(t)+\frac{q_{22}}{M_{2}}(m p h(t)-) g_{3}(t)\right)\left(M_{2}-g_{3}(t)\right)+a_{2} m b b(t) \\
\frac{d g_{4}(t)}{d t}=\left(p_{3}+\frac{q_{31}}{M_{3}} g_{4}(t)+\frac{q_{32}}{M_{3}}(m p h(t)-) g_{4}(t)\right)\left(M_{3}-g_{4}(t)\right)+a_{3} m b b(t)
\end{array}\right.
$$

В данной системе (1) $g_{i}(t)$ - доля пользователей технологии поколения $i$ в году $t$ в общей численности населения $(i=2,3,4), m p h(t)-$ доля пользователей мобильных телефонов в общей численности населения в год $t, \operatorname{int}(t)$ - прирост доли пользователей интернета в общей численности населения в году $\mathrm{t}$ по сравнению с годом $t-1, m b b(t)$ - прирост доли пользователей мобильного широкополосного доступа в интернет в общей численности населения в году $\mathrm{t}$ по сравнению с годом $t-1, p_{i}, q_{i j}$, $M_{i}, a_{i}$ - параметры модели для поколения $i-1$.

В связи с тем, что статистика по поколениям технологий мобильной связи является неполной по многим странам, панельные данные являются несбалансированными. Для расчета параметров модели был использован пакет Statistica, в котором оценка осуществлялась с помощью алгоритма Левенберга-Марквардта. Оценка параметров системы уравнений (1) была осуществлена с помощью приближения дифференциальных уравнений разностными, результаты оценивания представлены в табл. 2.

Таблица 2. Оценка параметров системы уравнений (1)

\begin{tabular}{|c|c|c|c|c|c|c|}
\hline \multirow{2}{*}{ Параметры } & \multicolumn{2}{|c|}{$2 \mathrm{G}$} & \multicolumn{2}{c|}{$3 \mathrm{G}$} & \multicolumn{2}{c|}{$4 \mathrm{G}$} \\
\cline { 2 - 7 } & значение & $\mathrm{t}$-stat & значение & t-stat & значение & t-stat \\
\hline $\mathrm{p}_{\mathrm{i}}$ & 0.27 & 3.8 & 0.56 & 4.0 & 0.001 & нет \\
\hline $\mathrm{q}_{\mathrm{i} 1}$ & 0.36 & 4.8 & -0.16 & -3.0 & 0.035 & 0.6 \\
\hline $\mathrm{q}_{\mathrm{i} 2}$ & -0.07 & -7.9 & -0.15 & -4.9 & 0.037 & 2.1 \\
\hline $\mathrm{M}_{\mathrm{i}}$ & 0.19 & 3.8 & 0.36 & 3.9 & 0.93 & 2.5 \\
\hline $\mathrm{a}_{\mathrm{i}}$ & 0.14 & 2.2 & 0.57 & 9.3 & 0.48 & 4.3 \\
\hline $\mathrm{R}^{2}$ & \multicolumn{2}{|c|}{0.51} & \multicolumn{2}{c|}{0.58} & \multicolumn{2}{c|}{0.42} \\
\hline $\mathrm{SSR}$ & 4.15 & \multicolumn{2}{c}{2.04} & \multicolumn{2}{c|}{0.33} \\
\hline
\end{tabular}

Анализ полученных результатов свидетельствует о наличии взаимного отрицательного влияния новых поколений технологий мобильной 
связи на предшествующие, т.е. вытеснение их с рынка. При этом отмечается рост рыночного потенциала каждого поколения технологий мобильной связи. Кроме того, выявлено положительное влияние распространения интернета на сети $2 \mathrm{G}$ и мобильного широкополосного доступа в интернет на сети $3 \mathrm{G}$ и $4 \mathrm{G}$. Наибольший коэффициент инновации $\left(p_{i}\right)$ получен для поколения $3 \mathrm{G}(0.65)$, имитации - для технологии $2 \mathrm{G}(0.36)$.

Учет патентных данных. Для исследования взаимного влияния поколений технологий мобильной связи была разработана модифицированная модель Лотки-Вольтерры с учетом патентных данных по поколениям технологий мобильной связи вида:

$$
\begin{aligned}
& \frac{d g_{2}(t)}{d t}=\left(a_{22} g_{2}(t)+a_{23} g_{3}(t)+a_{24} g_{4}(t)\right) g_{2}(t)+b_{1} \Delta p_{g_{2}}(t) c_{1} \Delta \operatorname{desk}(t) \\
& \frac{d g_{3}(t)}{d t}=\left(a_{32} g_{2}(t)+a_{33} g_{3}(t)+a_{34} g_{4}(t)\right) g_{3}(t)+b_{2} \Delta p_{g 3}(t) \\
& \frac{d g_{4}(t)}{d t}=\left(a_{43} g_{3}(t)+a_{44} g_{4}(t)\right) g_{4}(t)+b_{3} \Delta p_{l t e}(t)
\end{aligned}
$$

где $g_{2}, g_{3}, g_{4}$ - доля технологий $2 \mathrm{G}, 3 \mathrm{G}$ и $4 \mathrm{G}$ в общем количестве мобильных подключений в мире, $\Delta d e s k$ - прирост доли настольных компьютеров и ноутбуков в общем количестве устройств для выхода в интернет, $\Delta p_{g 2}$ и $\Delta p_{g 3}$ - прирост количества патентов по технологиям $2 \mathrm{G}$ и $3 \mathrm{G}$ соответственно, $\Delta p_{\text {lte }}$ - прирост количества патентов по технологии LTE за год.

Поиск соответствующих патентов был осуществлен в базе данных WIPO и проводился по ключевым словам «2G», «3G», «4G», «5G», «LTE», «gsm», «gprs». Система дифференциальных уравнений (2) была сведена к системе разностных уравнений. Полученные за период 20022018 гг. оценки параметров модели (2) представлены в табл. 3.

Таблица 3. Оценка параметров системы уравнений (2) за 2001-2018 гг. (по данным [19, 20, 21]).

\begin{tabular}{|c|l|c|c|c|c|c|c|}
\hline Поколения & Параметр & $\mathrm{a}_{\mathrm{i} 1}$ & $\mathrm{a}_{\mathrm{i} 2}$ & $\mathrm{a}_{\mathrm{i} 3}$ & $\mathrm{~b}_{\mathrm{i}}$ & $\mathrm{c}_{\mathrm{i}}$ & $\mathrm{R}^{2}$ \\
\hline \multirow{2}{*}{$2 \mathrm{G}$} & 3начение & 2.07 & -2.34 & -2.12 & 0.11 & 1.21 & \multirow{2}{*}{0.69} \\
\cline { 2 - 7 } & $\mathrm{t}$-stat & 2.7 & -2.9 & -3.1 & 2.3 & 2.7 & \\
\hline \multirow{2}{*}{$3 \mathrm{G}$} & 3начение & 0.57 & -0.34 & -1.31 & 0.05 & \multirow{2}{*}{ нет } & \multirow{2}{*}{0.73} \\
\cline { 2 - 7 } & t-stat & 2.4 & -3.1 & -3.6 & 2.5 & & \\
\hline \multirow{2}{*}{$4 \mathrm{G}$} & 3начение & -1.28 & 1.07 & -0.25 & 0.03 & \multirow{2}{*}{ нет } & \multirow{2}{*}{0.78} \\
\cline { 2 - 6 } & t-stat & -0.4 & 1.4 & -3.6 & 2.0 & & \\
\hline
\end{tabular}


В результате проведенных расчетов получено, что сети $3 \mathrm{G}$ и $4 \mathrm{G}$ являются «хищниками» по отношению к сетям предыдущего поколения ( $2 \mathrm{G}$ и $3 \mathrm{G}$ соответственно). В то же время между сетями $2 \mathrm{G}$ и $4 \mathrm{G}$ на рассматриваемом промежутке времени наблюдается конкуренция.

Прирост доли каждого поколения технологий мобильной связи положительно коррелирован с приростом числа патентов по соответствующей технологии (аналогичные результаты были получены для облачных вычислений [22]), прирост доли поколения $2 \mathrm{G}$ положительно коррелирован с приростом доли настольных компьютеров и ноутбуков в общем числе устройств, используемых для выхода в интернет. Однако доля таких устройств сокращается, они вытесняются смартфонами и планшетами с поддержкой технологий $3 \mathrm{G}$ и $4 \mathrm{G}$.

Заключение. В данной работе исследовано влияние социальноэкономических факторов на распространение поколений мобильных технологий $(2 \mathrm{G}, 3 \mathrm{G}$ и $4 \mathrm{G})$ в странах $\mathrm{OECD}$ и России за период 20002018 гг. Особое внимание было уделено конкуренции поколений и учету влияния накопленного количества патентов по каждому из них на уровень распространения поколения в мире. Выявлено наличие отрицательного влияния последующих поколений технологий мобильной связи на распространение предыдущих, при этом происходит рост рыночного потенциала технологий мобильной связи при переходе к новому поколению.

Выявлена положительная корреляция между уровнем распространения сетей $2 \mathrm{G}$ и приростом количества пользователей интернета, а также долей настольных компьютеров в общем количестве устройств для выхода в интернет. Для сетей $3 \mathrm{G}$ и $4 \mathrm{G}$ выявлена положительная корреляция их доли с приростом доли пользователей мобильного широкополосного доступа в интернет. Для всех поколений мобильных сетей получена положительная корреляция их прироста с приростом количества патентов по соответствующим технологиям. 


\section{СПИСОК ЛИТЕРАТУРЫ}

1. Варшавский A.E. Научно-технический прогресс в моделях экономического развития. - М.: Финансы и статистика, 1984.

2. Варшавский Л.Е. Моделирование динамики ключевых показателей рынков компонентов высокопроизводительных вычислительных систем // Tpyды ИСА РАН. 2017. Т. 67, №1. С.12-27.

3. Дубинина М.Г., Макарова Ю.А. Анализ технико-экономических показателей беспилотных транспортных средств // Научно-практический журнал "Конщепчии". 2018. №1(37). С. 28-44.

4. Pachauri A.K., Singh O. 5G Technology - Redefining wireless Communication in upcoming years // International Journal of Computer Science and Management Research. 2012. Vol. 1, Issue 1. P.12-19.

5. Gawas A.U. An Overview on Evolution of Mobile Wireless Communication Networks: 1G-6G // JRITCC. 2015. Vol. 3, Issue 5. P.3130-3133.

6. Ericsson Mobility Report. November 2017. URL: https://www.ericsson.com/en/mobility-report.

7. CNews. B России запущена первая промышленная сеть 5G. URL: https://www.cnews.ru/news/top/2020-01-27_v_rossii_zapushchena_pervaya.

8. Patel S., Shah D. 3G-4G Spectrum. 2011. URL: https://www.researchgate.net/publication/232614049 3G-4G

9. Fagbohun O.O. Comparative studies on $3 \mathrm{G}, 4 \mathrm{G}$ and $5 \mathrm{G}$ wireless technology // IOSR Journal of Electronics and Communication Engineering (IOSR-JECE). 2014. Vol.9, Issue 3, Ver. I. P. 88-94.

10. Technology Digest. Evolution of Mobile Communications (4G and 5G). Telecom Regulatory Authority of India, May 2018. URL: https://www.trai.gov.in/sites/default/files/TechnologyDigestMay2018.pdf

11. $4 \mathrm{G}$ service revenue to overtake $3 \mathrm{G}$ in 2016 . URL: https://telecoms.com/472922/4g-service-revenue-to-overtake-3g-in-2016/

12. Ерохин С.Д., Зайцева Ю.М. Анализ спектральной эффективности современных широкополосных систем связи. // Материалы VII Международной научно-технической конференции, 23-27 ноября 2010 г. С. 166-169.

13. The Mobile Revolution: How Mobile Technologies Drive a Trillion-Dollar Impact. The Boston Consulting Group, 2015.

14. The status of the $2 \mathrm{G} / 3 \mathrm{G}$ network sunset. URL: https://nae.global/en/the-statusof-the-2g-3g-network-sunset/

15. $2 \mathrm{G}$ осталось на обочине прогресса URL: http://www.comnews.ru/content/202599/2019-10-28/2019-w44/2g-ostalosobochine-progressa\#ixzz6Ap163jrx 
16. Cisco Visual Networking Index: Global Mobile Data Traffic Forecast Update, 2010-2015. URL: http://www.ieee802.org/3/ad_hoc/bwa/public/sep11/nowell_01_0911.pdf

17. Cisco Visual Networking Index (VNI) Complete Forecast Update, 2017-2022. URL: https://www.cisco.com/c/dam/m/en_us/network-intelligence/serviceprovider/digital-transformation/knowledge-networkwebinars/pdfs/1211_BUSINESS_SERVICES_CKN_PDF.pdf

18. Norton, J.A. and Bass, F.M. A Diffusion Theory Model of Adoption and Substitution for Successive Generations of High-Technology Products // Management Science. 1987. Vol.33, No.9. P.1069-1207. DOI: https://doi.org/10.1287/mnsc.33.9.1069

19. Counterclockwise: As $5 \mathrm{G}$ arrives we track the $3 \mathrm{G}$ and $4 \mathrm{G}$ adoption. URL: https://www.gsmarena.com/counterclockwise_new_networks_take_4_years_bef ore mainstream adoption-news-36661.php

20. StatCounter Global Stats. URL: https://gs.statcounter.com/platform-marketshare/desktop-mobile-tablet

21. WIPO IP Portal. Patentscope. URL: https://patentscope.wipo.int/search/en/search.jsf.

22. Дубинина М.Г. Анализ и моделирование диффузии облачных вычислений в России и за рубежом // Труды ИСА РАН 2017. Том 67, выпуск 4. С.22-34.

\section{MODELING DIFFUSION OF GENERATIONS OF MOBILE COMMUNICATION TECHNOLOGIES}

\section{Dubinina M.G.}

The paper is devoted to the analysis of technical and economic indicators of generations of mobile communication technologies, the identification of factors affecting their appearance and distribution. The diffusion models of $2 G$, $3 G$ and $4 G$ networks were developed for OECD countries and Russia for the period 2000-2018. Taking into account the influence of related technologies (the Internet and mobile broadband Internet access) and the mutual influence of previous and subsequent generations on the speed and scale of the spread of each type of network. 